\title{
Cognitive enhancement in schizophrenia with pharmacological interventions
}

\section{T Sharma*}

Address: Professor of Psychiatry, Director of the Clinical Neuroscience Research Centre, Dartford, Kent, United Kingdom

* Corresponding author

from International Society on Brain and Behaviour: Ist International Congress on Brain and Behaviour Hyatt Regency Hotel, Thessaloniki, Greece, 20-23 November, 2003

Published: 23 December 2003

Received: I November 2003

Annals of General Hospital Psychiatry 2003, 2(Suppl I):S35

This article is available from: http://www.general-hospital-psychiatry.com/content/2/SI/S35

Schizophrenia, the most severe form of psychopathology, affects about $1 \%$ in the general population. Cognitive impairment is a central feature of this illness. Most patients have a poor functional outcome, including deficits in social, occupational, and self-care activities. It is the most expensive psychiatric disorder to treat. The cost borne by the society in terms of social welfare administration and criminal justice, the time spent by unpaid caregivers, and the great loss of productivity due to the illness itself, are perhaps greater than the direct costs, such as, hospitalization. Functional deficits in schizophrenia are most strongly predicted by the current severity of cognitive impairment, followed by the severity of negative symptoms. Severity of positive symptoms is not strongly associated with the level of functional impairments, even in those with very poor outcome schizophrenia. There is thus an urgent need to find strategies for improving cognitive functioning in schizophrenia. It is widely felt by clinicians that conventional antipsychotic drugs have potent therapeutic actions on psychotic positive symptoms, but relatively weak actions on negative or cognitive symptoms. Atypical antipsychotics have been found to have greater effects on cognitive and negative symptoms than conventional antipsychotics, thus showing promise for cognitive enhancement and thus improved outcome of this illness possibly a result which may be due to the effects of 5HT and other neurotransmitter systems and normalisation of dopamine function by these compounds. Social cognition and learning are probably the domains most likely to have relevance to functional outcome. Facilitation of central cholinergic activity may form another potential treatment strategy for cognitive impairment in this population, since lesion and pharmacological studies in experimental animals and pharmacological probe studies in normal volunteers have repeatedly demonstrated a relationship between central cholinergic activity and cognitive functions, such as learning, memory and attention. Functional MRI (fMRI) is a non-invasive technique with good temporal and spatial resolution. It requires no radioactivity and offers the ability to map, almost in real-time, the physiological events occurring in the brain. fMRI can be used as a tool to map the longitudinal effects of antipsychotic drugs on the brain in schizophrenia. It allows us to carry out repeated measurements of cerebral neuronal activity and to investigate functional changes in the brain in treatment responders and non responders. It is thus possible to map the functional anatomy of neurocognitive improvement with atypical antipsychotics in schizophrenia. This presentation will outline new methods of brain imaging and how these methods may allow us to understand the long-term effects of cognitive improvement with antipsychotic drugs in schizophrenia 